\title{
On the integration of biotic interaction and environmental constraints at the biogeographical scale
}

\author{
Kévin Cazelles, Nicolas Mouquet, David Mouillot and Dominique Gravel
}

K. Cazelles (http:/lorcid.org/0000-0001-6619-9874) (kevin.cazelles@univ-montp2.fr) and D. Gravel, Dépt de biologie, chimie et géographie, Univ. du Québec à Rimouski, 300 Allée des Ursulines, QC G5L 3A1, Canada, and Québec Center for Biodiversity Science, Montreal, QC, Canada. - N. Mouquet and KC, Inst. des Sciences de l'Evolution, CNRS UMR 5554, Univ. de Montpellier, Place E. Bataillon, CC 065, FR-34095 Montpellier Cedex 5, France. - D. Mouillot, MARBEC (MARine Biodiverity Exploitation and Conservation), CC 093, Univ. de Montpellier, Place E. Bataillon, FR-34095 Montpellier Cedex 5, France, and Australian Research Council Centre of Excellence for Coral Reef Studies, James Cook Univ., Townsville, QLD 4811, Australia.

\begin{abstract}
Biogeography is primarily concerned with the spatial distribution of biodiversity, including performing scenarios in a changing environment. The efforts deployed to develop species distribution models have resulted in predictive tools, but have mostly remained correlative and have largely ignored biotic interactions. Here we build upon the theory of island biogeography as a first approximation to the assembly dynamics of local communities embedded within a metacommunity context. We include all types of interactions and introduce environmental constraints on colonization and extinction dynamics. We develop a probabilistic framework based on Markov chains and derive probabilities for the realization of species assemblages, rather than single species occurrences. We consider the expected distribution of species richness under different types of ecological interactions. We also illustrate the potential of our framework by studying the interplay between different ecological requirements, interactions and the distribution of biodiversity along an environmental gradient. Our framework supports the idea that the future research in biogeography requires a coherent integration of several ecological concepts into a single theory in order to perform conceptual and methodological innovations, such as the switch from single-species distribution to community distribution.
\end{abstract}

Biogeography is concerned with the description of the distribution of biodiversity and understanding its underlying processes. The discipline is central to the simulation of future scenarios of biodiversity under climate change (Thuiller et al. 2013). The extensive development of statistical models of species distributions based on actual ranges and environmental data have provided valuable knowledge and predictions (Kearney and Porter 2004), but often remain purely correlative. There is now consensus that future developments in biogeography will require solving critical limitations of species distribution models (Kissling et al. 2012) and incorporating explicitly biotic interactions and dispersal (Lavergne et al. 2010). This effort must be supported by theory in order to guide model development, maintain tractability and manage complexity. Developing a mechanistic theory of species distribution will require an integration of three fundamental principles and their interplay (Thuiller et al. 2013): 1) how local and regional dynamics are linked, 2) how species interact with the abiotic environment and 3) how they are embedded in a network of biotic interactions. Each of these principles are discussed in detail below.
A cornerstone of biogeography is the recognition of the contribution of regional-scale processes such as disturbances, historical contingencies (e.g. macro evolutionary history or glaciations) and dispersal limitations to local community dynamics (Ricklefs 1987). The metacommunity concept has been proposed as a simple framework to link different spatial scales in ecology (Leibold et al. 2004). It emphasizes reciprocal feedbacks between local scale processes, such as competitive interactions and local adaptation, and regional scale processes such as dispersal, gene flow, and speciation. A central concept of metacommunity ecology is the idea that local communities are highly dynamic owing to colonization events and local interaction, resulting in a spatial mosaic of assemblages sampled non-randomly from the regional species pool. As the concept matures there are new themes emerging, such as the investigation of evolution in metacommunities (Urban et al. 2008), and spatial food webs (Gravel et al. 2011, Massol et al. 2011). The field provides remarkable concepts and tools to build an integrated theory for biogeography.

Species distribution is also constrained by physiological requirements, which is at the core of the niche concept 
(Peterson 2011). The niche is usually defined as a $\mathrm{N}$-dimensional environmental and resource hyper-volume within which a species is able to maintain a viable population over the long term (Chase and Leibold 2003). Recent developments refined this definition based on demography and metapopulation dynamics (Holt and Barfield 2009). The abiotic niche, often referred as the Grinnelian niche, has been central to the development of species distribution models (SDMs, Jeschke and Strayer (2008)). Despite all of its criticisms, SDMs remain remarkably popular and operational for conservation ecology (Guisan et al. 2013). Recent attempts to improve the quantification of the niche include the addition of experimental assessments of the fundamental physiological constraints, as well as dispersal and proxies of biotic interactions (Boulangeat et al. 2012). The search for the most adequate set of environmental variables explaining diversity should be continued despite criticisms of the actual SDMs, and most of all must constitute a central principle of a general theory for biogeography.

Finally, species are not isolated, they are embedded within complex networks of ecological interactions. While interactions define community ecology, they are less informative for biogeography (Peterson 2003). Theory predicts that interactions in small community modules (2-4 species) should influence range limits (Gilman et al. 2010), but there is no extension to highly diverse communities. It has been hypothesized that factors determining distribution are hierarchical, such that climate would govern the distribution at the regional scale while biotic interactions would be more important at the local scale (Araújo and Rozenfeld 2014). However an increasing number of studies emphasizes the role of local interactions as a major factor influencing geographical ranges (Gotelli et al. 2010, Jabot and Bascompte 2012). The representation of interactions in a network is a convenient method to summarize the type and strength of interactions among species, their organization (Proulx et al. 2005) and their consequences on dynamics (Allesina and Tang 2012). Food webs were first considered in the development of a trophic theory of biogeography (Gravel et al. 2011), where it was shown that a diversity of interactions enhances persistence. Networks are however more than food webs and are rarely made of a single type of interaction (Kéfi et al. 2012). Mutualism, competition and indirect effects (Wootton 1994), for instance, also impact local environmental suitability (Godsoe and Harmon 2012). Tools and knowledge acquired through the study of local ecological networks, such as the community matrix and metrics of structure (Allesina and Tang 2012), must now be incorporated into a theory for biogeography.

These three principles should be all mixed together to provide an integrated assessment of their relative contribution to species distribution. To do so, the theory of island biogeography (hereafter referred as TIB) (MacArthur and Wilson 1967, Warren et al. 2015) is a convenient starting point. The TIB describes variations of species richness among islands as a dynamic equilibrium between two opposite processes, colonization and extinction, directly linked with island characteristics. The TIB is a metaphor that goes beyond the intrinsic interest of islands; it serves as a first approximation to understanding the assembly of local communities embedded in a metacommunity context with straightforward species flux. The simplicity of the model and the relevance of its predictions demonstrate after more than $50 \mathrm{yr}$ since its publication it is still a useful tool in ecology and conservation (Cook et al. 2002, Warren et al. 2015). The TIB emphasizes the role of regional processes to local community assembly. Indeed it can be regarded as the simplest representation of metacommunity dynamics (Leibold et al. 2004). Furthermore, the model is easily expandable. Following (Holt 2010, Gravel et al. 2011) introduced trophic interactions in the TIB (hereafter the trophic TIB, TTIB). Species interactions were found to be a key factor to understand species distributions, as the probability of finding any species in a locality increases with the generality of its diet and decreases with trophic rank.

We propose to generalize the TIB by integrating the three principles described above. The TIB already explicitly includes the effect of regional processes (colonization and extinction dynamics) on local community assembly, and the TTIB includes predator-prey interactions. We extend this framework to all potential interactions, thus resulting in a general model of metacommunity dynamics, akin to the Lotka-Volterra equations for local community dynamics. We also incorporate abiotic constraints on colonization and extinction dynamics. Hence we integrate the ingredients we believe are essential to model biodiversity distribution at the biogeographical scale. With this model in hand we then describe species distribution along environmental gradients. We use the mathematical formalism of Markov chains (Kemeny and Snell 1960, Black and McKane 2012) to derive expected assemblages and co-distribution at both the local and the regional scale. We illustrate how the interplay between biotic interactions and environmental requirements can affect the distribution of biodiversity over environmental gradients. Our results support the idea that the future research in biogeography require a consistent integration of several ecological concepts into a single framework to build promising approaches such as the switch from single-species distributions to community distributions.

\section{The model}

\section{A simple probabilistic biogeographical model}

The challenge of adding species interactions within the classical model of the TIB is gaining generality without losing simplicity. Following MacArthur and Wilson's theory, we model the dynamics of occurrence probability of a species $i$ in a local community. Species occurrence is the result of a balance between colonization and extinction dynamics, which occur at rates $c_{i}$ and $e_{i}$ respectively. Local species richness is given by the sum of occurrence probabilities over all species of the regional species pool, here simply defined as the set of all the $N$ species whose propagules (as defined in Simberloff and Wilson 1969) can land on the island considered. The model thereby takes into account local (extinction) and regional (colonization) processes. More precisely, the dynamics of occurrence probability of species i, $p_{i}$, follows:

$$
\frac{d p_{i}}{d t}=c_{i}\left(1-p_{i}\right)-e_{i} p_{i}
$$


Here, $c_{i}$ and $e_{i}$ are constant and a property of species $i$. In this widespread version of the TIB, also called the linear version of the TIB (Schoener 2010), the equilibrium occurrence probability of a species $i$ is given by $p_{i, e q}=\frac{c_{i}}{e_{i}+c_{i}}$. Also, species are assumed to be independent, therefore, the richness $S_{e q}$ is given by the sum of the $N$ different $p_{i, e q}$. The linear TIB can be modified to include trophic interactions (after Gravel et al. 2011) and we propose to extend it to all types of interactions. To reach that goal, the first step is to find a way to derive the expected species composition at any time. This composition can actually be depicted at any time by a vector of $N$ zeroes and ones indicating, respectively, presences and absences of each species considered, these combinations will be referred as assemblages. Following MacArthur and Wilson, we use a stochastic modelling approach to describe the dynamics of assemblages. The simplest scenario is the one species case. Here there are only two assemblages for the locality: one with species $i$ present and the other without. Let $X_{i}$ be a random variable describing the occurrence of species $i$. When species $i$ is present in the locality, $X_{i}$ is 1 , when it is absent $X_{i}$ is $0 ; X_{i}$ is then a Bernoulli variable. We define this random variable at any time $t$ which describes a stochastic process we denote $X_{i, t>0}$. The occurrence probability of species $i$ at time $t+d t$ ( $d t$ being a very small time step) is then given as follows:

$$
\begin{aligned}
P\left(X_{i, t+d t}=1\right)= & P\left(X_{i, t+d t}=1 \mid X_{i, t}=1\right) P\left(X_{i, t}=1\right) \\
& +P\left(X_{i, t+d t}=1 \mid X_{i, t}=0\right) P\left(X_{i, t}=0\right)
\end{aligned}
$$

$P\left(X_{i, \mathrm{t}+d t} \mid X_{i, t}\right)$ is the conditional probability describing $X_{i, t+d t}$ stating $X_{i, t}$. As $X_{i, t+d t}$ solely depends on $X_{i, t}$ (not on other earlier time steps) we have a discrete-time Markov chain. In this process, species $i$ will be present in a locality at time $t+d t$ if it was already present at time $t$ and persisted (meaning it did not go extinct) with probability $\left(1-e_{i} d t\right)$, or if it was absent and colonized the community from the mainland (with probability $c_{i} d t$ ). Note that $d t$ is small enough to get $0<c_{i} d t<1$ and $0<e_{i} d t<1$. Hence, Eq. (2) becomes:

$$
P\left(X_{i, t+d t}=1\right)=c_{i} d t P\left(X_{i, t}=0\right)+\left(1-e_{i} d t\right) P\left(X_{i, t}=1\right)
$$

This equation leads to when $d t$ tends to zero. This formulation keeps the simplicity of the original MacArthur and Wilson model, but can also more generally be used to consider the probability of any given assemblage. $P\left(X_{i, t+d t} X_{i, t}\right)$ defines the rules to switch from one assemblage to one another during the interval $d t$. There are $N$ occurrence probabilities we gather within $Y_{t>0}=\left(X_{1, t>0}, X_{2, t>0}, \ldots, X_{P, t>0}\right)$ which leads to the description of $2^{N}$ assemblages depicted by a given collection of zeros and ones. The conditional probabilities provide the transition from one local assemblage $k$ to any other $l$ during $d t$. For any species $i$ there are only four possible cases: at time $t$ either species $i$ is locally absent and colonizes the locality $\left(I_{1}\right)$ or not $\left(I_{2}\right)$ during $d t$, either species $i$ is present and goes extinct $\left(I_{3}\right)$ or survives $\left(I_{4}\right)$ during $d t$. The conditional probabilities between two communities states $(l$ and $k)$ can then be simply derived from these four probabilities:

$$
\begin{aligned}
& P\left(Y_{t+d t}=\text { state }^{\prime} \mid Y_{t}={ }^{\prime} \text { state } l '\right)=\prod_{i_{1} \in I_{1}} c_{i_{1}} d t \prod_{i_{t} \in I_{2}}\left(1-c_{i_{2}} d t\right) \\
& \prod_{i_{3} \in I_{3}} e_{i_{3}} d t \prod_{i_{4} \in I_{4}}\left(1-e_{i_{4}} d t\right)
\end{aligned}
$$

We now apply the complete probability formula as defined in to get the probability of observing one assemblage at $t+d t$ given its state at $t$. This is where the main benefit of Markov chain models is: it allows us to derivate exact solutions for the probabilities for assemblages, instead of a set of independent occurrence probabilities for each species. This approach is promising for building joint species distribution models (see Discussion). This property will be fully explored in the next section to include interactions.

Consider as an example a pool of two species $(N=2)$ for which we find four assemblages: at any time $t$, a locality can contain either two species $\left(\mathrm{X}_{1, t}=1, \mathrm{X}_{2, t}=1\right)$, only one species $\left(\mathrm{X}_{1, t}=1, X_{2, t}=0\right)$ and $\left(\mathrm{X}_{1, t}=0, \mathrm{X}_{2, t}=1\right)$, or none of them $\left(\mathrm{X}_{1, t}=0, X_{2}=0\right)$. The transition from one local assemblage to another is then easily obtained. Table 1 presents these conditional probabilities. This is actually the transition matrix of a Markov chain we solve (by calculating one eigen value, see below). To illustrate the dynamics expected in TIB from our assemblage point of view, we simulate the model as follows: $c_{1}=c_{2}=0.15, e_{1}=e_{2}=0.05, P\left(X_{1,0}=0\right.$, $\left.X_{2,0}=0\right)=0.6$ and $P\left(X_{1,0}=1, X_{2,0}=0\right)=0.4$, so species 2 is absent at time $t=0$. Just as for the single species situation, the probabilities of observing each community tend to an equilibrium (Fig. 1A). By summing the previous probabilities where a given species ( 1 or 2 ) is present (the conditional probabilities) we get its overall occurrence probability (marginal probability, Fig. 1B). Finally, we can calculate the expected number of species in a locality (Fig. 1C), in agreement with the TIB. Interestingly, this calculation is often achieved in the other way. Firstly, the presence probability of all species are computed: $P\left(X_{i}\right)=\frac{c_{i}}{c_{i}+e_{i}}$. Then the richness is obtained under the assumption that species are independent and so $P\left(X_{i}, X_{j}\right)=P\left(X_{i}\right) P\left(X_{j}\right)$. We show below that occurrence probabilities of each assemblage is a key to introduce interactions among species.

Table 1. Conditional probabilities between potential assemblages. At any time $t$ we calculate all the possible conditional probabilities between the four potential assemblages for a two species regional pool. These probabilities are derived by multiplying probabilities of single species events defined in Eq. (4). By doing so, we build the transition matrix of our Markov chain where species are assumed to be independent. We release this hypothesis by linking extinction coefficients and species assemblages.

\begin{tabular}{lllll}
\hline & \multicolumn{3}{c}{$\left(X_{1, t+d t} X_{2, t+d t}\right)$} \\
\cline { 2 - 5 }$\left(X_{1, t}, X_{2, t}\right)$ & \multicolumn{1}{c}{$(0,0)$} & $(0,1)$ & $(1,0)$ & $(1,1)$ \\
\hline$(0,0)$ & $\left(1-c_{1} d t\right)\left(1-c_{2} d t\right)$ & $\left(1-c_{1} d t\right) c_{2} d t$ & $c_{1} d t\left(1-c_{2} d t\right)$ & $c_{1} d t c_{2} d t$ \\
$(0,1)$ & $\left(1-c_{1} d t\right) e_{2} d t$ & $\left(1-c_{1} d t\right)\left(1-e_{2} d t\right)$ & $c_{1} d t e_{2} d t$ & $c_{1} d t\left(1-e_{2} d t\right)$ \\
$(1,0)$ & $e_{1} d t\left(1-c_{2} d t\right)$ & $\left(1-c_{1} d t\right) e_{2} d t$ & $\left(1-e_{1} d t\right)\left(1-c_{2} d t\right)$ & $\left(1-e_{1} d t\right) c_{2} d t$ \\
$(1,1)$ & $e_{1} d t e_{2} d t$ & $\left(1-e_{1} d t\right) e_{2} d t$ & $\left(1-e_{1} d t\right)\left(1-e_{2} d t\right)$ \\
\hline
\end{tabular}


(A)

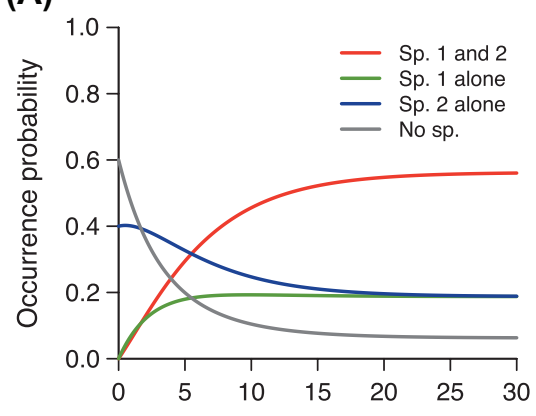

(B)

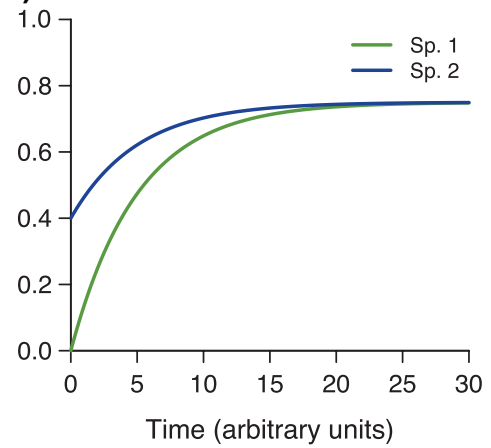

(C)

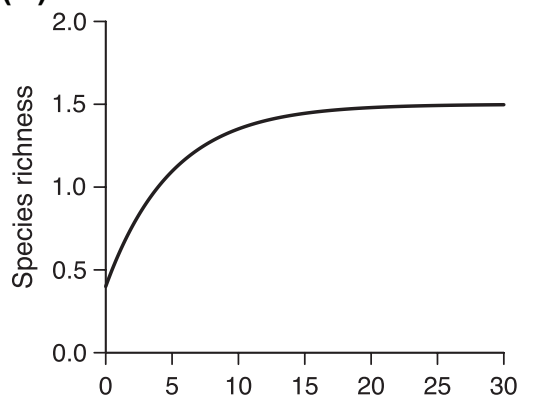

Figure 1. Dynamics of the community assembly. As a direct development of MacArthur and Wilson (1967) model, we simulate for two species, the dynamics of the four communities states with different initial conditions associated (A). By summing every states where one given species is present we get the occurrence probability of two considered species (B). Finally by summing the four states probabilities weighted by their species richness, we get the classical model of MacArthur and Wilson (C). The calculation of (B) and (C) does not require species being independent while classical approaches focus on (B) to derive (A) and (C) under this assumption of independence.

\section{Integrating biotic interactions}

We start by representing the interaction network by a community matrix $A$ of $N$ species that we incorporate into the Markovian TIB chain model. The elements $\alpha_{i, j}$ of $A$ quantify the effect of species $j$ on the dynamics of species $i$. We first consider that interactions could alter both the colonization and the extinction probabilities (Gravel et al. 2011). When $\alpha_{i j}$ is negative, the colonization probability of species $i$ decreases and/or its extinction probability increases when $j$ is found locally. Inversely, when $\alpha_{i, j}$ is positive, the colonization probability increases and/or the extinction probability decreases. Note that diagonal elements provide the extinction probability per time unit when no other species is present.

The elements of the community matrix $A$ represent the pairwise effects of ecological interactions on transition probabilities. To account for the cumulative effects of local interactions on transition probabilities, we make colonization and extinction probabilities community dependent. As explained above, at a time $t$, the $Y_{t}$ vector gives the local assemblages. We calculate the sum of interactions at any time and for each species as $v=A Y_{t}^{T}$ (where $T$ denotes the transpose operator). Our approach can be interpreted as a spatial analogue to the generalized Lotka-Volterra model because it takes into account the impact of the whole network of interactions on each species dynamics and can deal with any type of interaction. We denote the coefficients of $v$ by $v_{i}$, they are species-specific parameters (weighted by parameter $d_{i}$ ) of two species-specific functions: $f_{i}$ and $g_{i}$, respectively, standing for extinction and colonization probabilities for species $i$. Note that at this stage we do not define any specific function relating interactions to colonization $\left(f_{i}\right)$ and extinction probabilities $\left(g_{i}\right)$, to keep the description of the model general (see below for some proposed functions). At each time step, the local community composition impacts: 1) the colonization probability of species present in the regional pool but absent from the local community, and 2) the extinction probability of species present on the local community.
If we expand the two species example (labeled 1 and 2, Table 1), according to the general model, we define two $f$ functions $\left(f_{1}\right.$ and $\left.f_{2}\right)$ linking interaction and extinction and two $g$ functions linking interaction and colonization $\left(g_{1}, g_{2}\right)$. At this stage, to reduce the model's complexity, we consider that interactions solely impact extinction probabilities. This assumption is reasonable if we consider that local interactions impact mostly demography (possibly leading to extinction) and that colonization success solely depends on the first propagule (interactions occur after arrivals). Therefore $g_{1}$ and $g_{2}$ are constant functions, respectively, returning $c_{1}$ and $c_{2}$. The functions $f$ are assumed to have a sigmoid shape. There are many reasons such a function is of interest: 1 ) we get a clear link with the basic extinction probability, i.e. $e_{i}$ for an interaction strength of $0 ; 2$ ) we define both a minimum and a maximum extinction probability; 3 ) the first interactions to occur are the most influential (Gravel et al. 2011 considered that at least one interaction was required to persist, which is very similar).

$$
\begin{aligned}
& f_{i}(v)=e_{i, \text { min }} \\
& \quad+\frac{1}{\frac{1}{e_{i, \text { max }}-e_{i, \text { min }}}+\left(\frac{1}{e_{i}-e_{i, \text { min }}}-\frac{1}{e_{i, \text { max }}-e_{i, \text { min }}}\right) \exp \left(d_{i} * v_{i}\right)} \\
& g_{i}(v)=c_{i}
\end{aligned}
$$

To illustrate how interactions modify occurrence probabilities, we simulate the model for two networks: $A_{1}$ where all interactions are negative and $A_{2}$ where they are all positive. We consider null diagonal elements for both networks. Consequently, there is no difference with the model without interaction when one species is alone in the locality. Simulation results are presented at Fig. 2. Panel A presents the functions $f_{1}$ and $f_{2}$ we chose for our two species example. For networks $A_{1}$ and $A_{2}$, we show how interactions alter the probabilities of observing different assemblages (respectively, Fig. 2B and Fig. 2C). The assemblage with both species present (solid red lines) is the most affected by interactions, switching from an occurrence probability of 0.2 (for negative 
(A)

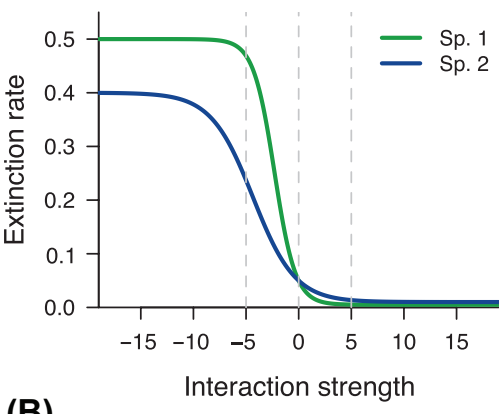

(B)

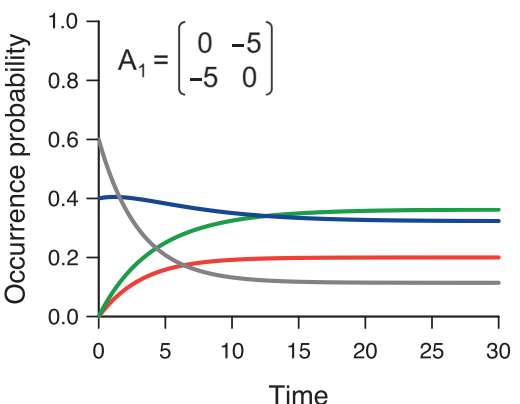

(D)

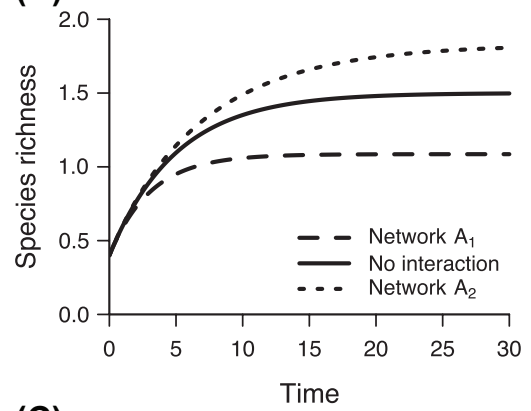

(C)

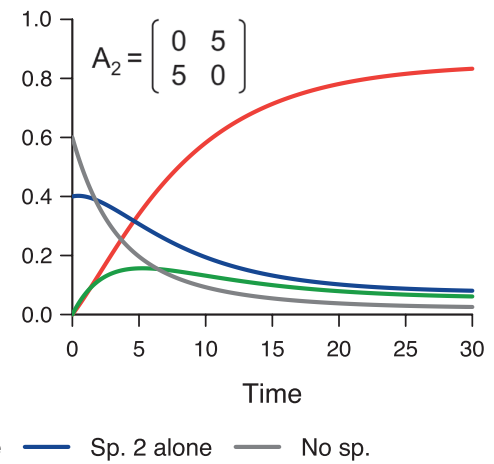

Figure 2. Effects of biotic interactions on colonization-extinction dynamics. For any species $i$, the extinction probability $e_{i}$ is related to the strength of the interaction as shown in (A). The intersections of extinction curves with the grey dotted lines indicate the potential values of $e_{i}$ according to the different biotic context $\left(A_{1}, A_{2}\right.$ and without interaction). We set the other parameters as follows: $c_{1}=c_{2}=0.15$, $P\left(X_{1,0}=1, X_{2,0}=0\right)=0.4, P\left(X_{1,0}=0, X_{2,0}=0\right)=0.6, P\left(X_{1,0}=0, X_{2,0}=1\right)=P\left(X_{1,0}=1, X_{1,0}=1\right)=0$. We then simulate the model for two simple networks $A_{1}$ and $A_{2}$ and present community assembly dynamics associated ((B) and $\left.(\mathrm{C})\right)$. Finally we compare the expected species richness on the locality (D) for our two networks and for the case without interaction which corresponds to the widespread linear version of the TIB.

interactions) to 0.8 (for positive interactions). Positive interactions enhance, as expected, co-occurrence while negative interactions prevent species from being found on the same island. Consequently, occurrence probabilities of single species states are lower in $A_{2}$ than in $A_{1}$. According to a defined network, occurrence probabilities of the different assemblages are then modified, which affect the expected species richness (Fig. 2D).

\section{Integrating environmental gradients}

We now introduce the effect of abiotic conditions, such as climatic variables, on transition probabilities. We denote the vector of $n$ environmental conditions by $w: w=\left(w_{1}\right.$, $\left.w_{2}, \ldots w_{n}\right)$. We first assume that physiological constraints can affect both colonization and extinction probabilities through the functions $f_{i}$ and $g_{i}$ (affecting, respectively, extinction and colonization rates). Again the model in its general formulation does not presume any shape for these functions. We now have all the ingredients of an integrated model of biogeography as the transition probabilities at a location depend on 1) species-specific colonization and existence probabilities, 2) the network of interactions, 3) local community composition, and 4) local environmental conditions. In the general formulation of the model, functions $f_{i}$ and $g_{i}$ are functions of multiple variables $(v$ and $w$ ).

At any time $t$, for a regional pool of $N$ species among which interactions are summarized by the community matrix
$A$, in an environment characterized by $w$, we can derive all transition probabilities. These constitute a transition matrix of a Markov chain that we denote $M(v, w)$. Its elements, $\mu_{k, l}$ $(v, w)$, give the probability the locality in assemblage $k$ turns into assemblage $l$ (left side of Eq. (4)):

$$
\begin{array}{r}
\mu_{k, l}(v, w)= \\
\prod_{i_{i} \in I_{1}} g_{i_{1}}(v, w) d t \prod_{i_{i} \in I_{2}}\left(1-g_{i_{2}}(v, w) d t\right) \\
\prod_{i_{3} \in I_{3}} f_{i_{3}}(v, w) d t \prod_{i_{4} \in I_{4}}\left(1-f_{i_{4}}(v, w) d t\right)
\end{array}
$$

Note that the dimension of $M(v, w)$ will increase as a power of the number of species $N$ and thus can rapidly becomes large. Let $C_{t}$ be the line vector of the probability of observing each assemblage, defined by: $C_{t}=\left(P\left(Y_{t}=\right.\right.$ 'state 1'), $P\left(Y_{t}=\right.$ 'state 2 ' $), \ldots, P\left(Y_{t}=\right.$ 'state $\left.\left.2^{\mathrm{N}}\right)\right)$. The Markov chain formalism defines the probability of the future community composition at time $t+d t$ as $C_{t+d t} M=C_{t} M$ asymptotically reaches the $C_{e q}$ after a certain number of time steps. $C_{e q}$ is given by the normalized left eigenvector associated to the first left eigenvalue.

$\lim _{\substack{l \rightarrow+\infty \\ l \in N}} C_{0} M^{l}=C_{e q}$

$C_{e q}$ contains the probability of all assemblages at the equilibrium. The occurrence probability of a given species, is provided by the sum of all probabilities of assemblage where that species is present. The richness at the equilibrium $S_{e q}$ is the sum of $C_{e q}$ elements weighted by the number of species found in the associated assemblages. 
For the sake of illustration, we further reduce the complexity of our model. We have previously removed the interactions $(v)$ from colonization $(g)$ functions; we now state that extinction does not depend on environmental variables and so we remove the abiotic environment $(w)$ from extinction functions $(f)$. This can be interpreted as the effects of the abiotic environment on extinction rate being included within $e_{\mathrm{i}}$ (i.e. extinction rate without interaction). Furthermore, we assume solely one environmental variable and a Gaussian shape for $g_{i}$ functions. A simple function with a clear optimum and very low colonization for extreme environment

values is.
$g_{i}\left(w_{1}\right)=g\left(w_{1},\left(c_{i}, h_{i}, r_{i}\right)\right)=c_{i} * \exp \left(-\left(\frac{w_{1}-h_{i}}{r_{i}}\right)^{2}\right)$

This enables us to define an environmental optimum $\left(h_{i}\right)$, a colonization probability per time unit $\left(c_{i}\right)$ and also suitable range $\left(r_{i}\right)$ for each species. Figure 3 presents the interplay between the three components of the integrated biogeographical model. The chosen functions for the environmentcolonization relationship are illustrated in panel A. For the two previous networks ( $A_{1}$ and $A_{2}$; illustrated in Fig. 2) we now compute the probabilities of observing the different assemblages at equilibrium, along the environmental gradient (panel $\mathrm{B}$ and $\mathrm{C}$ ). When interactions are negative (network $A_{1}$ ), species repulse each other and rarely co-occur, whatever the environment is. Most of their occurrence follow their abiotic niche (blue and green lines) as they are barely found together. Inversely, when interactions are positive (for $A_{2}$ network) they often co-occur where their abiotic niches overlap, thereby decreasing the probability of an empty community (panel D, solid grey line). Finally, we present how interactions modify the resulting community composition along the environmental gradient (panel D). Species richness is constrained by the distribution of abiotic niches and the sign of the interactions. As expected, the role of interactions is strongest when abiotic niches largely overlap.

\section{Exploring the model}

In our exploration, we choose a regional pool $N$ of 10 species to keep the number of assemblages reasonable $\left(2^{10}=1024\right)$ and to numerically compute the exact solution of the equilibrium distribution $C_{e q}$. We consider four types of interaction matrices $A$. The first situation corresponds to the classical MacArthur and Wilson model, where the $A$ matrix is null (no interactions). For the three other scenarios we generate random matrices with fixed connectance (number of existing links divided by the number of potential links). The coefficients within $A$ are drawn uniformly within $[0,1]$ and the sign of the interaction is determined by the action of one species on another, for instance, a predator has a negative impact on its prey leading to a negative $\alpha$ coefficient; in return, a prey has a positive effect on its predators. The intensity of the interaction is then determined by the $d$ coefficient of extinction functions (Eq. (8)). We assume that the distribution of the links are given by the niche model (Williams and Martinez 2000). This model is simple and provides relevant random food webs with the same
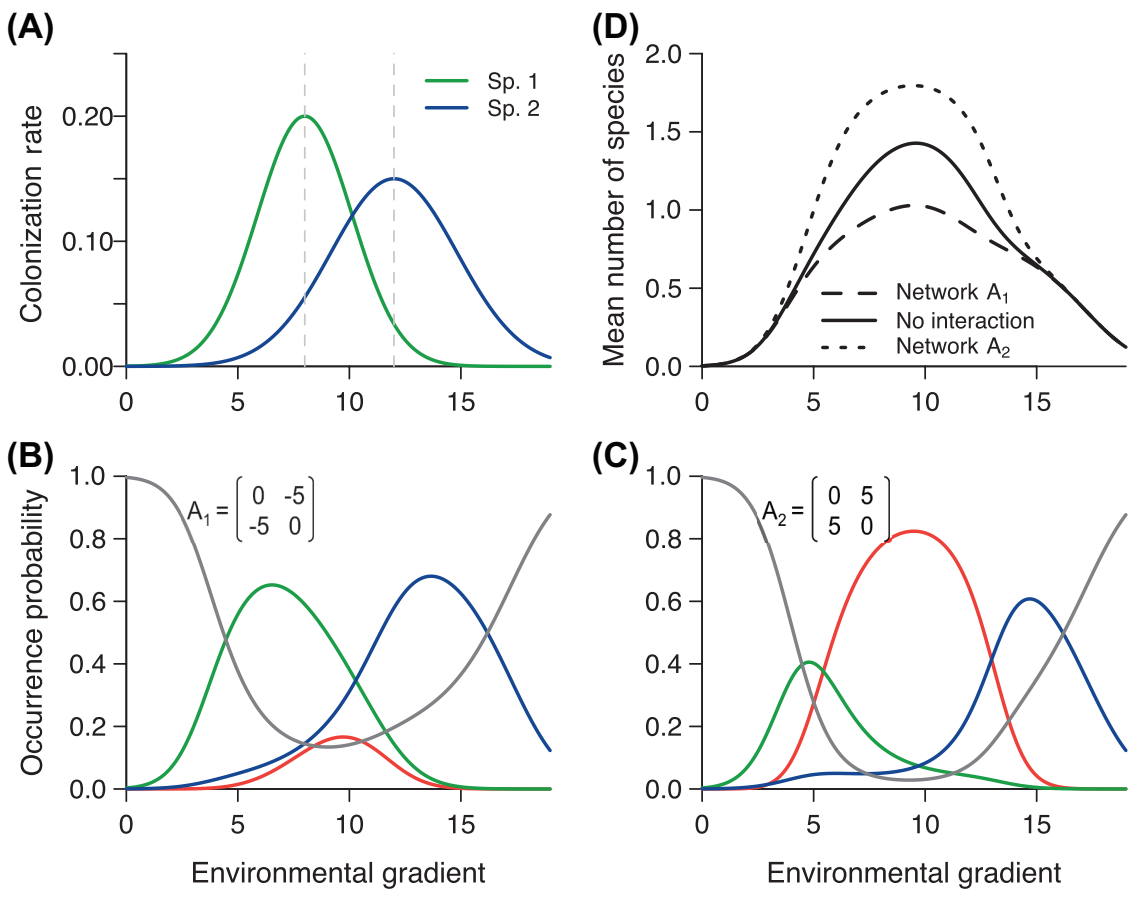

(C)

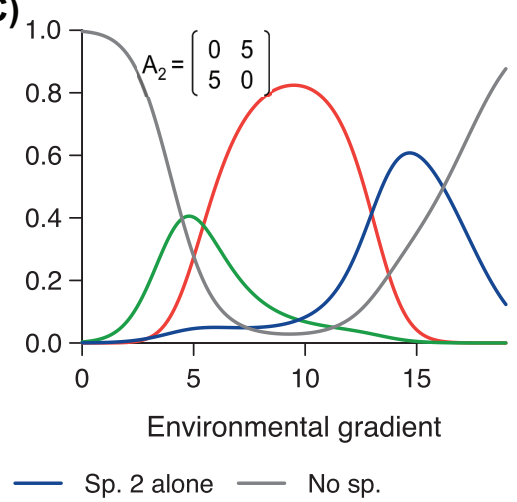

Figure 3. Equilibrium for interacting species along an environmental gradient. The colonization probability of species $i, c_{i}$, is related to the environment variable $w$ according to species-specific requirements (A). The intersection of the colonization curve of species $i$ with the grey dotted lines represents the value of $c_{i}$ associated with its environmental optimum $h_{i}$. We compute equilibrium occurrence probabilities for the different assemblages along the environmental gradient, for the networks $A_{1}$ with negative interactions $(\mathrm{B})$ and $A_{2}$ with positive one (C). We calculate the expected species richness on the locality for the two networks and without interaction (D). 
number of positive and negative interactions. For the two last scenarios, we keep the rules to distribute the links, but turn all the coefficients in $A$ positive to generate a mutualism network, or negative for competition networks. Although these basic structures with exclusive interaction types are not realistic, they facilitate comparison among results. Hence, the scenarios simply differ by the sign distribution within the matrix $A: 1)$ no interaction $A$ is null, 2) predation mixes both signs ' $+/-$ ', 3) mutualism only ' + ', 4) competition, only '-'. With these scenarios in hands, we 1) present the assemblages probabilities associated with a given level of species richness and 2) we look at the species richness expected along an environmental gradient. For all figures presented hereafter we used 1000 randomly-generated $A$ matrices.

\section{Assemblage probabilities}

First, we illustrate how interactions affect richness of species assemblages. To do so, we build the Markov chains for all the $1000 \mathrm{~A}$ matrices generated (connectance set to 0.2 ) and we calculate the vector $C_{e q}$. This is a vector of 1024 occurrence probabilities (as we consider 10 species). Then we sum all the probabilities that correspond to assemblages of the same richness. We do so for three values of $d$ coefficient $(0.1,1$ and $10)$; that is, we look at how the strength of interaction affect community richness predictions. Figure 4 presents the results of such investigation, with panels $\mathrm{A}$ to $\mathrm{C}$ corresponding to the results for the three different values of the $d$ parameter.

As expected, positive interactions increase local species richness by diminishing extinction probabilities, while negative interactions weaken large communities (see the contrast between blue and red symbols on Fig. 4). This is stressed as interaction strengths increase, that is for increasing values of $d$. Indeed, when $d$ is low, there is almost no difference among scenarios because interactions do not impact strongly colonization and extinction dynamics; occurring species can be regarded as mostly independent. All scenarios converge to the classical TIB scenario (no-interaction, grey symbols), the resulting species richness distribution is binomial (here for all species $p_{i, e q}=0.5$ as $\left.\mathrm{c}_{i}=\mathrm{e}_{i}=10^{-5}\right)$. Differences between interaction types increase with $d$. Species rich mutualistic communities are more likely to occur since positive interactions tends to promote co-occurrence. Therefore species occurrence can be dramatically affected by the strength of interactions: for $d=10$ (panel C in Fig. 4), the species richness is 9.46 for positive interactions (red symbols), 2.24 for the negative ones (blue symbols) and 5 without interactions. When positive and negative interactions are mixed (our predation scenario, green symbols on Fig. 4), it seems that the negative effect of predators on their prey prevails and so predation reduces species richness, but less than for competitive networks.

As we introduce variability through the use of randomlygenerated matrices, we also compute the standard deviation associated with occurrence probabilities. The variability is provided as the coloured vertical bars found in Fig. 4 which stand for $50 \%$ of the total standard deviation. Clearly, variability increases with 1) the strength of interaction and 2) the occurrence probability. Although this can simply reflect the variability of values found in $A$ matrices, this could potentially be caused by the variability of the location of non-zero values in $A$ matrices; that is, the structure of the networks we use.

\section{Biodiversity distribution over environmental gradients}

In this section, we introduce an environmental gradient to emphasize the interplay between interactions species-specific requirements along an environmental gradient. Our environmental gradient takes values from 0 to 30 , for each of them we calculate the expected species richness associated to all scenario. To do so, we start by computing the colonization functions ( $g_{i}$ functions): species optima $h_{i}$ are drawn
(A)

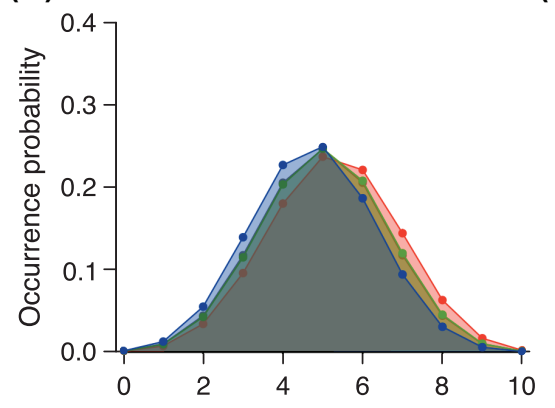

(B)

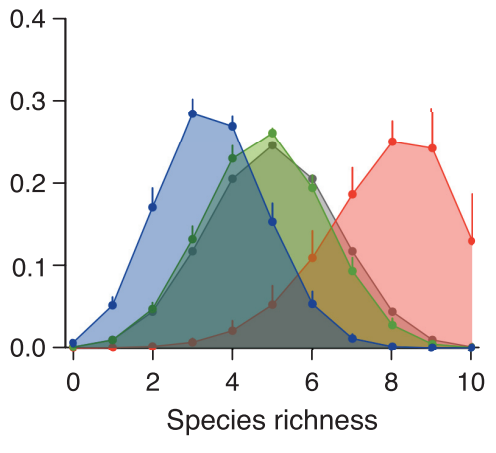

(C)

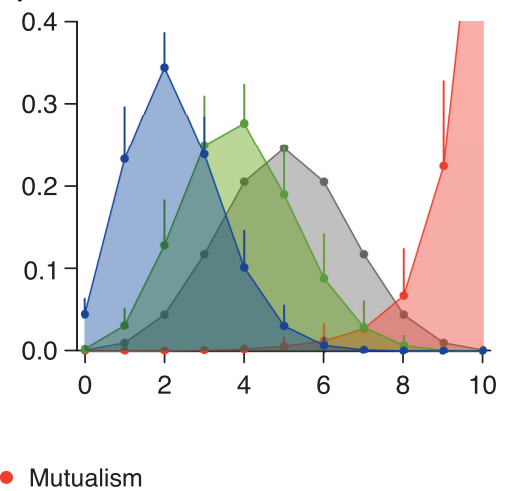

Figure 4. Probabilities of species richness for different types of interaction. We compute expected species richness at the equilibrium with the following set of parameters: $\left.e_{i}=10^{-5}, e_{i, \min }=10^{-3} e_{i}, e_{i, \max }\right)=10^{3} e_{i}$ and $c_{i}=10^{-5}$. We do so for three different interaction weights: $d=0.1$ (A), $d=1(\mathrm{~B}), d=10(\mathrm{C})$. In each panel, the four colours stand for the following types of networks: no interaction (grey), predation (green), competition (blue) and mutualism (red). Probabilities associated to coloured points are the means calculated for 1000 interaction matrices randomly-generated according to the niche model (Williams and Martinez 2000) with a connectance set to 0.2. Additionally, vertical bars represent $50 \%$ of the standard deviations associated to these means. To facilitate comparisons among panels, we do not represent the occurrence probability of the 10 assemblages community in panel $\mathrm{C}$ for mutualism, which is 0.66 (the standard deviation associated is 0.33 ). 
from a uniform distribution from the range $[10,20]$ and the widths of the abiotic niches are kept constant for all the simulations $r_{i}=5$. Then we build the Markov chains for the different values of the environmental gradient and for the different $A$ matrices. Again, we derive the vector $C_{e q}$ and we sum its elements, i.e. occurrence probabilities of assemblage community, weighted by the species richness to which they refer. We repeat the procedure for an increasing value of connectance of $A$ matrices: from 0 to 0.4 . For this section, the parameter $d$ is set to 10 , also extinction parameters are set as follows: $e_{i}=10^{-5}, e_{i \text { min }}=10^{-3} e_{i}, e_{i, \max }=10^{3} e_{i}$ and $c_{i}=10^{-5}$. Like so we obtain the profile of species richness we report on Fig. 5.

For all scenarios, the richness is maximal at the center of the environmental gradient (Fig. 5). This is due to the distribution of species optima in the range $[10,20]$. Also this is the range of environmental values for which the effect of interaction are the most important. Indeed, the higher the colonization probabilities, the higher interactions occur, therefore, interactions strongly impact species richness for favourable abiotic conditions. We also find that changes in species richness increase with connectance, as depicted by the colour of the solid lines for the three panels of Fig. 5: from black (without interaction) to the lightest blue (connectance set to 0.4$)$.

Species richness is inversely related to connectance when interactions are negative (panel A in Fig. 5). Moreover, when abiotic conditions are favourable, the number of species expected tends to 1 . At the centre of the gradient, even though colonization probabilities are maximal, many species colonize but likely go extinct because of competition. We expect the locality to be most often occupied by species that are not affected by competition. Alternatively, in the case of positive interactions (panel B in Fig. 5), the expected species richness is strongly enhanced by interactions even for low connectance. The expected species richness tends to reach the total number of species from the most favourable to semi-harsh abiotic conditions. As the connectance increases the Gaussian shape of the richness profile turns into a hat shape, which has one major consequence: from favourable to semi-harsh conditions, the species richness is maintained thanks to positive interaction, but it also quickly collapses as the environment becomes slightly harsher.

Finally, when positive and negative interactions are mixed, the higher the connectance, the flatter the richness profile (panel B in Fig. 5). The expected species richness declines as connectance increases but far less than it does for negative interactions only. We think this is caused by the colonization of numerous prey that promote the survival of predators which in turn prevent assemblages to be as large as they can be without interaction (as predators reduce the persistence of prey). Conversely, from harsh to intermediate environmental conditions, mixed sign interactions positively affect the species richness. We explain this as the consequence of the benefit predators take from the preys presence. Assemblages with few predators, promoted by positive effect of the prey on their predators, may be relatively stable. Since colonization is low, this assemblage may enhance species richness over time but they may also collapse as soon as an extra predator colonizes the island.

\section{Discussion}

Understanding how colonization-extinction dynamics influence species distribution and community structure remains a major challenge in biogeography (Wiens 2011, Godsoe and Harmon 2012, Jabot and Bascompte 2012). Here, we build upon the simplicity of the theory of island biogeography (TIB) to integrate crucial ecological processes, namely biotic and abiotic dimensions of the niche. Using the formalism of Markov chains, we derive an exact general solution for the occurrence probabilities of all possible assemblages that we calculate numerically (up to 10 species). Our approach is in stark contrast to the classic TIB (MacArthur and Wilson 1967) where environmental gradients were not introduced and the co-occurrence among species was not modelled, despite empirical evidence of their impact (Diamond and

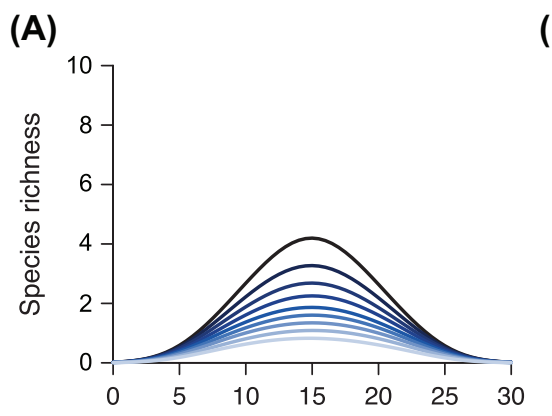

(B)

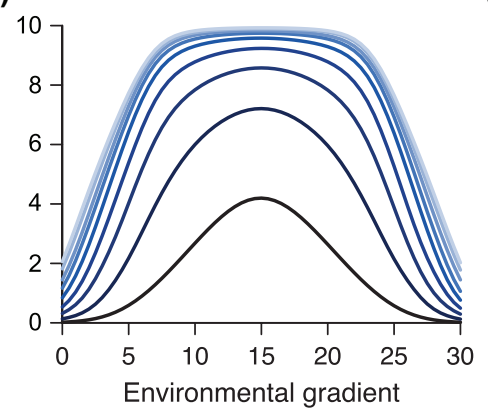

(C)

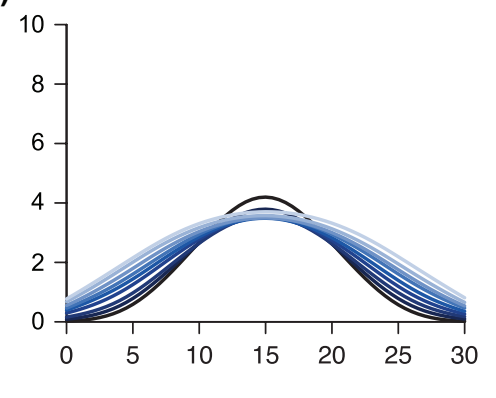

Connectance:

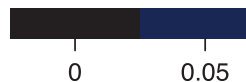

0

0.05

0.1

0.15

0.2

0.25

0.3

Figure 5. Biodiversity distribution along environmental and connectance gradients. We compute the expected species richness along an environmental gradient for competition (A), mutualism (B) and predation (C). We do so for different values of connectance depicted by the shades of blue. Species richness profile associated with the scenario without interaction is provided in each panel by the darkest solid line (connectance set to 0 ). Abiotic niches do have the same range for all species $\left(r_{i}=5\right)$ and the optima are randomly drawn in the interval $[10,20]$. The interaction weight $(d)$ is set to 10 . The extinction parameters are set as follows: $e_{i}=10^{-5}, e_{i, m i n}=10^{-3} e_{i}, \mathrm{e}_{i, \max }=10^{3} e_{i}$ and $c_{i}=10^{-5}$. 
Gilpin 1982). By taking these constraints together we reveal how they interplay and affect species richness. We believe our approach offers new perspectives on the theory of biogeography and will support the development of species distribution models with the addition of species interactions.

In our model, we introduce the effect of biotic interactions as an ecological process affecting colonization/extinction probabilities. This has already been considered in many ways in the literature. For instance, more than forty years ago, Levins and Culver introduced extinction and migration rates affected by competition and showed analytically how it reduces co-occurrence (Levins and Culver 1971). More recently, Jabot and Bascompte introduced production of eggs and seeds affected by interaction in an individualbased, meta-community framework and, hence, highlighted the potential effects of interactions on local diversity (Jabot and Bascompte 2012). Also, Calcagno and colleagues demonstrated that tuning extinction and colonization rates based on the trophic relationships among species could explain the limited length of food chain (Calcagno et al. 2011). In contrast with previous studies, our approach is fully rooted on the TIB which yields well-defined null predictions (adding neither interaction nor environmental gradients), focuses on assemblages, and allows the investigation of the impact of any kind of network, including mixed interactions.

Networks are convenient representations of the structure of ecological communities to study persistence and resilience (Thébault and Fontaine 2010). A strength of our model is that it not only takes all direct interactions into account, but also indirect ones (Wootton 1994). For instance, in a linear trophic chain of three species, the occurrence of the top predator depends not only on the presence of its prey but also on the species at the bottom of the chain (Gravel et al. 2011). This means that the distribution of the top predator will be influenced not only by its own abiotic requirements, but also by those of its prey and the species at the bottom of the chain. The signature of such indirect interactions should be common in co-occurrence networks. This property comes from the assumption that interactions change extinction rates and the Markov chain formalism employed. Our formalism therefore provides a tool, similar to the general Lotka-Volterra equations for the local scale, that could be used to study the emergence of indirect interactions in networks at the large spatial scale.

The challenge of developing joint species distribution models (Pellissier et al. 2013, Pollock et al. 2014) have recently motivated researchers to investigate co-occurrence (Veech 2013, Araújo and Rozenfeld 2014). Our framework helps to disentangle the two main processes by which nonrandom species associations (co-occurrence) can arise. First, two species not interacting with each other could be nonrandomly co-distributed because of similar or antagonistic ecological requirements. As we introduced an abiotic constraint on the colonization probability, some assemblages will be more likely than others on a given environment simply because some species are favoured and others filtered out. We thus expect to find a signature of the covariance in species response to the environment on these assemblage probabilities. Secondly, non-random co-distribution will arise from ecological interactions. We considered an additive impact of all ecological interactions a species is experiencing from the community. Species interact in various ways, but at the end all interactions do impact demography by definition. This reality enters the model by either enhancing of decreasing extinction probabilities. In other words, the occurrence of a single species is derived from the expectation of observing all other species in the community.

Our framework therefore provides a formalism to investigate the relationship between co-occurrence networks (Araújo et al. 2011) and interaction networks. There is a significant amount of information contained in the data of co-occurrence, which is overlooked by most current methods of community analysis. Standard species distribution models are fitted to univariate presence/absence data, neglecting the information contained in the distribution of associated taxa. Multivariate statistics summarize the spatial structure of ecological communities, but they are essentially limited to the description of co-occurrence, they are not meant to predict species distributions conditional on other species. Most analyses of co-occurrence aggregate pairwise observations into a single index for the whole community, thereby missing substantial information pertaining to the consequences of biotic interactions (Boulangeat et al. 2012). This situation is not surprising given there is no general theory for co-occurrence despite recent efforts (Cazelles et al. 2015). Current hypotheses are mostly limited to negative interactions, leading to negative co-occurrence (repulsion), or positive interactions, leading to positive co-occurrence (attraction). Many theoretical achievements are required to study co-occurrence for more complex assemblages, mixing positive, negative and antagonistic interactions. In addition, the impact of indirect interactions emerging in interaction networks on species distribution is ignored. Our approach provides a formal framework to overcome these limitations as we calculate assemblage probability at biogeographical scale and then derive co-occurrence. It also allows the decomposition of the strength of pairwise associations between abiotic and biotic drivers, opening the way for novel statistical developments of species distribution models taking into account this multi-occurrence information. We propose that studying the role of biotic factors at large scale requires us to introduce them as assemblages instead of adding species as factors which likely leads to non-equivocal conclusion (Araújo and Luoto 2007). In addition, our approach is not limited to species pairs, the assemblage probabilities provide a valuable tool to the co-occurrence of groups of species such as motifs (Stouffer et al. 2007).

The importance of interactions across different scales is still debated (McGill 2010, Araújo and Rozenfeld 2014). A common assumption is that interactions are negligible at large spatial scales, based on the rational that abiotic filters primarily determine the composition of assemblages (Pearson and Dawson 2003). This argument persists even though theoretical (Gravel et al. 2011) and empirical (Gotelli et al. 2010) evidence suggest the opposite. The key issue to solve this debate is thus to know how interactions can influence species assemblages with increasing spatial scale. Although the TIB still provide insights into the assembly of natural communities, the success of recent approaches integrating interactions strongly support their relevance at large scales. Indeed the addition of network structure (Pellissier et al. 2013) or correlation between species (Pollock et al. 2014) 
as proxies for interactions have adequately improved forecast accuracy. Here we do not solve this fundamental issue, however our model illustrates how species distribution at large scale will be impacted by the kind of interaction, their numbers and their distribution.

Although our framework is not readily applicable to real datasets, it nonetheless provides a theoretical foundation for the derivation of new statistical modelling approaches. We propose a different perspective which is rooted on theory, in contrast with what is usually done with phenomenological model representing the structure of the data (Thuiller et al. 2013). There are nonetheless significant challenges to apply our framework to empirical data. First, we must find a way to deal with large numbers of species. At present, given $N$ species in the regional pool, we compute an eigen vector of $2^{n}$ probabilities from a $2^{N}{ }^{*} 2^{N}$ transition matrix of a Markov chain. Moreover, in its current formulation, it requires us to evaluate a very large amount of data including a description of network of the same species across time and space to get accurate estimations. Solving this issue will requires a rational to reduce the number of species considered. This could be achieved either by inference of the relevant interactions, or alternatively by pooling species into groups. A systematic and rigorous method to build meaningful groups of interacting species from proxies such as traits and phylogenies remains to be developed, but there are nonetheless promising avenues (Baskerville et al. 2011). The relatively small number (from 3 to 7 ) of dimensions to ecological networks, i.e. the number of trait-axes required to properly infer interactions (Eklöf et al. 2013), supports its feasibility. A second challenge is to account for spatial structure that constrains population flux. Despite the theoretical developments, applied approaches to model species distribution struggle to introduce it efficiently (Boulangeat et al. 2012). The islandmainland approximation remains elegant but might be too simple for applied situations. One solution may be to identify source and sink localities (Boulangeat et al. 2012). This requires us to consider 1) species abundances and 2) spatial structures which would strongly increase the complexity of the model. One first step forward could be to apply the Levins model rather than the island-mainland model as Levins and Culver did to study the impact of competition (Levins and Culver 1971).

Despite our call for a new integrated theory of biogeography, we acknowledge the limitations of the framework. Recent studies aimed at integrating population dynamics, for instance, using approximations from the metabolic theory of ecology. This is a hopeful direction to assess local extinction risk, accounting for network structure, body size and abundance (Schneider et al. 2012). Beyond body size, other functional traits (McGill et al. 2006) could help us to escape from species singularity toward more general rules. Hence, approximating food web structure could be done using traits (Gravel et al. 2013) and energetic requirements can be easily quantified through body size and local temperature constraints (Brown et al. 2004). Moreover considering changes in traits over time may be a key to introduce evolutionary processes. This would help us to release one strong assumption of our work: no speciation processes are taken into account. Although it might not matter for short time periods, having a pool of species unchanged becomes a major issue for time scales that exceed by far the lifespans of species we consider. Further, our framework could be applied to investigate diversification dynamics on remote areas, with a particular emphasis on the effect of ecological interactions on adaptive radiations. Despite the complexity of such model, it would very likely provide valuable insights on the future of biodiversity under current global change.

Since the seminal work of Davis et al. (1998), there is growing evidence that the response of species to climate change must be studied at the community scale (Suttle et al. 2007). Even though species respond individually to climate change, they are constrained by complex direct and indirect biotic interactions emerging from large scale organization (Lavergne et al. 2010). The study of Cahill et al. (2013) has revealed the difficulties to link climate changes and species extinction. Even when the climate is expected to drive local extinctions, it actually implies a chain of perturbations amidst which biotic factors prevail (e.g. loss of prey Durance and Ormerod 2010). For instance, species contributing to the persistence of plant-pollinator networks are paradoxically the most vulnerable to extinction (Saavedra et al. 2011), highlighting the risk of extinction cascades. As Säterberg et al. expressed, 'the species to be the first to go extinct is not the one whose mortality rate is increased but instead some other species in the food web', thereby suggesting that perturbations which affect species differently also spread over the network making extinction difficult to predict (Säterberg et al. 2013). Although this is fully understandable as species interact, this makes forecasting of future species distributions more complicated. Therefore the challenge of proposing biodiversity scenarios to global change requires new approaches integrating ecological processes over time and spatial scales, and to disentangle their relative contribution (Lavergne et al. 2010). We think that the assemblage-based approach we propose here is a promising perspective to introduce interactions in biogeographical models.

Acknowledgement - We thank Chantal L. Hutchison for insightful comments on the manuscript. KC was supported by a grant from the Ministry of Higher Education and Research of France. DG was supported by a NSERC Discovery grant and the Canadian Research Chair program. NM was supported by the CNRS.

\section{References}

Allesina, S. and Tang, S. 2012. Stability criteria for complex ecosystems. - Nature 483: 205-208.

Araújo, M. B. and Luoto, M. 2007. The importance of biotic interactions for modelling species distributions under climate change. - Global Ecol. Biogeogr. 16: 743-753.

Araújo, M. B. and Rozenfeld, A. 2014. The geographic scaling of biotic interactions. - Ecography 37: 406-415.

Araújo, M. B. et al. 2011. Using species co-occurrence networks to assess the impacts of climate change. - Ecography 34: 897-908.

Baskerville, E. B. et al. 2011. Spatial guilds in the Serengeti food web revealed by a Bayesian group model. - PLoS Comput. Biol. 7: e1002321.

Black, A. J. and McKane, A. J. 2012. Stochastic formulation of ecological models and their applications. - Trends Ecol. Evol. 27: 337-345. 
Boulangeat, I. et al. 2012. Accounting for dispersal and biotic interactions to disentangle the drivers of species distributions and their abundances. - Ecol. Lett. 15: 584-593.

Brown, J. H. et al. 2004. Toward a metabolic theory of ecology. - Ecology 85: 1771-1789.

Cahill, A. E. et al. 2013. How does climate change cause extinction? - Proc. R. Soc. B 280: 20121890.

Calcagno, V. et al. 2011. Constraints on food chain length arising from regional metacommunity dynamics. - Proc. R. Soc. B 278: 3042-3049.

Cazelles, K. et al. 2015. A theory for species co-occurrence in interaction networks. - Theor. Ecol. <http://dx.doi. org/10.1007/s12080-015-0281-9>.

Chase, J. M. and Leibold, M. A. 2003. Ecological niches: linking classical and contemporary approaches. - Univ. of Chicago Press.

Cook, W. M. et al. 2002. Island theory, matrix effects and species richness patterns in habitat fragments. - Ecol. Lett. 5: 619-623.

Davis, A. J. et al. 1998. Making mistakes when predicting shifts in species range in response to global warming. - Nature 391: 783-786.

Diamond, J. M. and Gilpin, M. E. 1982. Examination of the "null" model of connor and simberloff for species co-occurrences on islands. - Oecologia 52: 64-74.

Durance, I. and Ormerod, S. J. 2010. Evidence for the role of climate in the local extinction of a cool-water triclad. - J. North Am. Benthol. Soc. 29: 1367-1378.

Eklöf, A. et al. 2013. The dimensionality of ecological networks. - Ecol. Lett. 16: 577-583.

Gilman, S. E. et al. 2010. A framework for community interactions under climate change. - Trends Ecol. Evol. 25: 325-331.

Godsoe, W. and Harmon, L. J. 2012. How do species interactions affect species distribution models? - Ecography 35: 811-820.

Gotelli, N. J. et al. 2010. Macroecological signals of species interactions in the Danish avifauna. - Proc. Natl Acad. Sci USA 107: 5030-5035.

Gravel, D. et al. 2011. Trophic theory of island biogeography. - Ecol. Lett. 14: 1010-1016.

Gravel, D. et al. 2013. Inferring food web structure from predator-prey body size relationships. - Methods Ecol. Evol. 4: 1083-1090.

Guisan, A. et al. 2013. Predicting species distributions for conservation decisions. - Ecol. Lett. 16: 1424-1435.

Holt, R. D. 2010. Toward a trophic island biogeography. - In: Losos, J. B. and Ricklefs, R. E. (eds), The theory of island biogeography revisited. Princeton Univ. Press, pp. 143-185.

Holt, R. D. and Barfield, M. 2009. Trophic interactions and range limits: the diverse roles of predation. - Proc. R. Soc. B 276: 1435-1442.

Jabot, F. and Bascompte, J. 2012. Bitrophic interactions shape biodiversity in space. - Proc. Natl Acad. Sci. USA 109: 4521-4526.

Jeschke, J. M. and Strayer, D. L. 2008. Usefulness of bioclimatic models for studying climate change and invasive species. - Ann. N. Y. Acad. Sci. 1134: 1-24.

Kearney, M. and Porter, W. P. 2004. Mapping the fundamental niche: physiology, climate and the distribution of a nocturnal lizard. - Ecology 85: 3119-3131.

Kéfi, S. et al. 2012. More than a meal... integrating non-feeding interactions into food webs. - Ecol. Lett. 15: 291-300.

Kemeny, J. G. and Snell, J. L. 1960. Finite markov chains. - Springer.

Kissling, W. D. et al. 2012. Towards novel approaches to modelling biotic interactions in multispecies assemblages at large spatial extents. - J. Biogeogr. 39: 2163-2178.

Lavergne, S. et al. 2010. Biodiversity and climate change: integrating evolutionary and ecological responses of species and communities. - Annu. Rev. Ecol. Evol. Syst. 41: 321-350.
Leibold, M. A. et al. 2004. The metacommunity concept: a framework for multi-scale community ecology. - Ecol. Lett. 7: 601-613.

Levins, R. and Culver, D. 1971. Regional coexistence of species and competition between rare species. - Proc. Natl Acad. Sci. USA 68: 1246-1248.

MacArthur, R. H. and Wilson, E. O. 1967. Theory of island biogeography. - Princeton Univ. Press.

Massol, F. et al. 2011. Linking community and ecosystem dynamics through spatial ecology. - Ecol. Lett. 14: 313-323.

McGill, B. J. 2010. Ecology. Matters of scale. - Science 328: 575-576.

McGill, B. J. et al. 2006. Rebuilding community ecology from functional traits. - Trends Ecol. Evol. 21: 178-185.

Pearson, R. G. and Dawson, T. P. 2003. Predicting the impacts of climate change on the distribution of species: are bioclimate envelope models useful? - Global Ecol. Biogeogr. 12: 361-371.

Pellissier, L. et al. 2013. Combining food web and species distribution models for improved community projections. - Ecol. Evol. 3: 4572-4583.

Peterson, A. T. 2003. Predicting the geography of species' invasions via ecological niche modeling. - Q. Rev. Biol. 78: 419-433.

Peterson, A. T. 2011. Ecological niche conservatism: a time-structured review of evidence. - J. Biogeogr. 38: 817-827.

Pollock, L. J. et al. 2014. Understanding co-occurrence by modelling species simultaneously with a joint species distribution model (JSDM). - Methods Ecol. Evol. 5: 397-406.

Proulx, S. R. et al. 2005. Network thinking in ecology and evolution. - Trends Ecol. Evol. 20: 345-353.

Ricklefs, R. E. 1987. Community diversity: relative roles of local and regional processes. - Science 235: 167-171.

Saavedra, S. et al. 2011. Strong contributors to network persistence are the most vulnerable to extinction. - Nature 478: 233-235.

Säterberg, T. et al. 2013. High frequency of functional extinctions in ecological networks. - Nature 499: 468-470.

Schneider, F. D. et al. 2012. Body mass constraints on feeding rates determine the consequences of predator loss. - Ecol. Lett. 15: 436-443.

Schoener, T. W. 2010. The MacArthur-Wilson equilibrium model. - In: Losos, J. B. and Ricklefs, R. E. (eds), The theory of island biogeography revisited. Princeton Univ. Press, pp. 52-87.

Simberloff, D. S. and Wilson, E. O. 1969. Experimental zoogeography of islands: the colonization of empty islands. - Ecology 50: 277-296.

Stouffer, D. B. et al. 2007. Evidence for the existence of a robust pattern of prey selection in food webs. - Proc. R. Soc. B 274: 1931-1940.

Suttle, K. B. et al. 2007. Species interactions reverse grassland responses to changing climate. - Science 315: 640-642.

Thébault, E. and Fontaine, C. 2010. Stability of ecological communities and the architecture of mutualistic and trophic networks. - Science 329: 853-856.

Thuiller, W. et al. 2013. A road map for integrating eco-evolutionary processes into biodiversity models. - Ecol. Lett. 16: 94-105.

Urban, M. C. et al. 2008. The evolutionary ecology of metacommunities. - Trends Ecol. Evol. 23: 311-317.

Veech, J. A. 2013. A probabilistic model for analysing species cooccurrence. - Global Ecol. Biogeogr. 22: 252-260.

Warren, B. H. et al. 2015. Islands as model systems in ecology and evolution: prospects fifty years after MacArthur-Wilson. - Ecol. Lett. 18: 200-217.

Wiens, J. J. 2011. The niche, biogeography and species interactions. - Phil. Trans. R. Soc. B 366: 2336-2350.

Williams, R. J. and Martinez, N. D. 2000. Simple rules yield complex food webs. - Nature 404: 180-183.

Wootton, J. T. 1994. The nature and consequences of indirect effects in ecological communities. - Annu. Rev. Ecol. Evol. Syst. 25: 443-466. 